\section{Ácido úrico: una molécula con acciones paradójicas en la insuficiencia cardiaca}

\author{
HERNÁN ALCAÍNO ${ }^{1}$, DOUGLAS GREIG ${ }^{2}$, \\ PABLO CASTRO ${ }^{2}$, HUGO VERDEJO ${ }^{2}$, \\ ROSEMARIE MELLADO ${ }^{3}$, LORENA GARCÍA ${ }^{4}$, \\ GUILLERMO DÍAZ-ARAYA ${ }^{4}$, CLARA QUIROGA ${ }^{4}$, \\ MARIO CHIONG ${ }^{4}$, SERGIO LAVANDERO $^{4,5}$
}

\section{The role of uric acid in heart failure}

Complications and mortality of heart failure are high, despite the availability of several forms of treatment. Uric acid, the end product of purine metabolism would actively participate in the pathophysiology of heart failure. However, there is no consensus about its action in cardiovascular disease. Serum uric acid would have a protective antioxidant activity. This action could help to reduce or counteract the processes that cause or appear as a result of heart failure. However, these protective properties would vanish in the intracellular environment or in highly hydrophobic areas such as atherosclerotic plaques and adipose tissue. This review discusses the paradoxical action of uric acid in the pathophysiology of heart failure.

(Rev Med Chile 2011; 139: 505-515).

Key words: Heart failure; Oxidative stress; Uric acid.

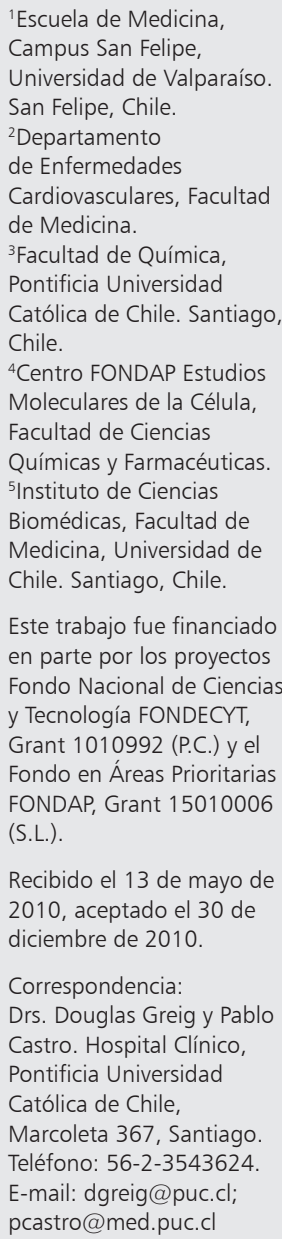

A pesar de los avances en el estudio de la fisiopatología y terapia de la insuficiencia cardiaca, esta patología continúa siendo una de las principales causas de hospitalización y mortalidad tanto a nivel nacional como mundial ${ }^{1-3}$.

Actualmente, la insuficiencia cardiaca (IC) se entiende como un proceso clínico muy complejo, caracterizado por un inadecuado funcionamiento ventricular y una serie de alteraciones neurohormonales que impiden al corazón suplir las necesidades metabólicas mínimas de los tejidos periféricos, traduciéndose en el establecimiento de estrés oxidativo e inflamación ${ }^{4}$.

Sólo en los últimos años se ha estudiado la importancia pronóstica y terapéutica de los trastornos metabólicos a nivel cardiovascular ${ }^{5-7}$. Entre éstos, ha adquirido alto interés el estudio de las alteraciones del metabolismo del ácido úrico ${ }^{8,9}$. Hasta la fecha, no existe un consenso acerca del papel del ácido úrico en la IC. Por una parte, diversos estudios básicos y clínicos sugieren que participa activamente en su génesis y progresión ${ }^{10-18}$, mientras otras investigaciones postulan una acción beneficiosa del ácido úrico a través de una actividad antioxidante ${ }^{19-25}$.

Esta revisión discute cómo esta molécula puede ser tanto deletérea como benéfica. Para ello, se describirá la biología del ácido úrico, se presentarán y analizarán sus efectos deletéreos y beneficiosos $y$, finalmente, se sugieren algunos mecanismos que permiten explicar su acción paradójica en el sistema cardiovascular. 


\section{Biología del ácido úrico}

El ácido úrico es el producto final del catabolismo de las purinas en humanos producido mediante la acción enzimática de la xantino óxidorreductasa $(\mathrm{XOR})^{26}$ (Figura 1). Esta enzima se descubrió en la leche y, desde un principio, se pensó que podría participar activamente en la producción de especies reactivas del oxígeno $(\mathrm{EROs})^{27}$. Existe en dos formas que son convertibles entre sí, la xantino oxidasa $(\mathrm{XO})$ y la xantino deshidrogenasa $(\mathrm{XDH})^{28}$. La XO reduce oxígeno molecular, mientras que la XDH reduce tanto oxígeno como el $\mathrm{NAD}^{+}$teniendo una gran afinidad por el segundo sustrato ${ }^{27,28}$. Además, la XDH es más abundante in vivo ${ }^{29}$ y puede ser convertida a XO en forma irreversible por una variedad de enzimas tales como tripsina, quimiotripsina y pancreatina $^{30}$. El hígado y el intestino delgado son las mayores fuentes de $\mathrm{XO}^{31}$, pero actualmente existe evidencia que tanto el corazón como el endotelio vascular expresan $\mathrm{XO}^{32-34}$. De hecho, su actividad se ha podido determinar a nivel endotelial humano, denominándose XO extracelular o unida al endotelio $(\mathrm{ecXO})^{35}$. La principal acción enzimática de la XO es la conversión catalítica consecutiva de hipoxantina a xantina y luego desde xantina a ácido úrico. Paralelamente, como subproductos de estas reacciones, se for- man potentes EROs, moléculas que poseen alta reactividad con otros sustratos (Figura 1), tales como peróxido de hidrógeno $\left(\mathrm{H}_{2} \mathrm{O}_{2}\right)$ y anión superóxido $\left(\mathrm{O}_{2}{ }^{-}\right)^{26-28}$. Por otro lado, el ácido úrico es principalmente excretado por los riñones y su concentración plasmática depende del pH de la orina, como también de otros factores tales como el volumen de orina, el volumen corporal, la función renal, dieta y el uso de ciertos medicamentos, entre otros ${ }^{21,36}$. Los humanos poseen niveles más altos de ácido úrico que la mayoría de los otros mamíferos, pues estos últimos poseen una enzima llamada uricasa o urato oxidasa que metaboliza al ácido úrico circulante, produciendo alantoína que finalmente se elimina por la orina ${ }^{37}$. El gen que codifica para la uricasa es inactivo en humanos y primates. De hecho, se piensa que su inactivación ocurrió en la época del mioceno ( 8 a 20 millones de años atrás), mediante la mutación en la región promotora de este gen, conduciendo a la pérdida de su actividad. Diversos estudios han sugerido que esta mutación ha conferido una ventaja de sobrevivencia a los humanos y primates, pues se postula que se logra mantener la presión sanguínea en ambientes carentes de sal. Otra hipótesis es que un aumento del ácido úrico por esta mutación, permitió incrementar la inteligencia a través de propiedades de estimulación cerebral que tendría el ácido úrico ${ }^{37}$.

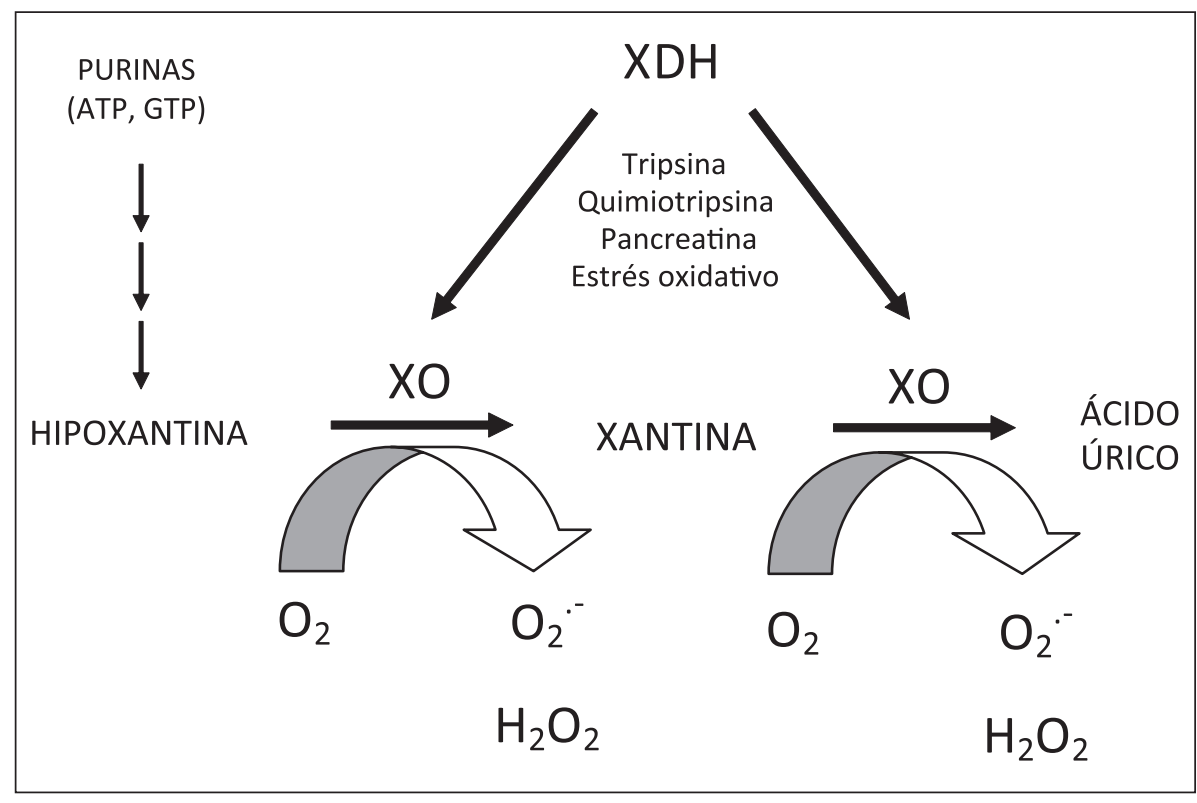

Figura 1. Metabolismo del ácido úrico en humanos. 
Rol del ácido úrico en insuficiencia cardiaca - H. Alcaíno et al

\section{Efectos deletéreos del ácido úrico en la insufi- ciencia cardiaca}

\section{Estudios básicos}

La posible asociación entre la sobreproducción de ácido úrico, el daño miocárdico y la disfunción endotelial parece ser debido a la sobreregulación de la actividad de XO, la cual es uno de los sistemas enzimáticos in vivo de mayor producción de EROs $^{38}$.

Se han descrito varios mecanismos por la cual el ácido úrico podría ser deletéreo en la insuficiencia cardiaca. Se ha demostrado que el ácido úrico producido en células dañadas, como en el caso de células necróticas, produce daño celular mediado por el sistema inmune ${ }^{39}$, aumento en la producción del factor de necrosis tumoral tipo alfa $(\mathrm{TNF}-\alpha)$ estimulada por endotoxina ${ }^{40}$, oxidación del colesterol LDL ${ }^{41}$ y la producción de citoquinas pro-inflamatorias ${ }^{42,43}$.

A nivel vascular, el ácido úrico estimula la síntesis de la proteína quimioatractante de monocitos tipo 1 (MCP-1) y proteína C reactiva (PCR), la cual estimula la infiltración de macrófagos e incrementa la actividad plaquetaria, pudiendo elevar el riesgo de trombosis ${ }^{44,45}$. Ambos fenómenos, se asocian a inflamación y disfunción endotelial, los cuales contribuyen al desarrollo de insuficiencia cardiaca $^{46,47}$

Modelos experimentales sugieren que la hiperuricemia participa en la génesis de enfermedades cardiovasculares, incluida la insuficiencia cardiaca. Mazzali et al describieron que ratas tratadas con un inhibidor de la uricasa desarrollaron hipertensión al cabo de varias semanas. En este modelo, se comprobó que la hipertensión se debió a la vasoconstricción renal mediada por el ácido úrico proveniente de la reducción de los niveles endoteliales de óxido nítrico, con la activación subsecuente del sistema renina-angiotensina ${ }^{48}$. Posteriormente, en estas ratas hiperuricémicas se desarrolla enfermedad renal microvascular con histología similar a la arterioesclerosis, característica de la hipertensión esencial. El hecho de que las alteraciones microvasculares se desarrollen aun cuando la presión arterial esté controlada mediante un diurético, junto con la demostración de los efectos directos del ácido úrico sobre las células endoteliales y las células del músculo liso vascular ${ }^{49,50}$ indica que el ácido úrico puede causar microvasculopatía independientemente de hipertensión arterial.

\section{Estudios clínicos y epidemiológicos}

Diversos estudios clínicos y epidemiológicos han comprobado la relación entre los niveles de ácido úrico y varias enfermedades cardiovasculares, como la hipertensión arterial ${ }^{51,52}$, síndrome metabólico ${ }^{53,54}$, enfermedad coronaria ${ }^{55,56}$, preeclampsia $^{57}$, enfermedad renal ${ }^{58,59}$ e insuficiencia cardiaca aguda $^{17}$ y crónica ${ }^{13,16-18,60}$. Al respecto, la relación entre ácido úrico e insuficiencia cardiaca ha sido observada a partir de niveles plasmáticos relativamente normales de ácido úrico $(>5,2$ $\mathrm{mg} / \mathrm{dL}$ ) y no sólo en aquellos pacientes con hiperuricemia reconocida (niveles de ácido úrico $>6 \mathrm{mg} / \mathrm{dL}$ en mujeres y $>7 \mathrm{mg} / \mathrm{dL}$ en hombres). Un estudio en 212 pacientes con insuficiencia cardiaca aguda y fracción de eyección ventricular izquierda (FEVI) $<40 \%$, mostró que la presencia de hiperuricemia ajustada por sexo, se asoció a un mayor riesgo de muerte o de rehospitalización en el largo plazo (mediana de seguimiento de 2 años), independiente de la capacidad funcional y presencia de insuficiencia renal previa. Estos resultados fueron significativos luego del ajuste por otras variables como la edad, función renal, clase NYHA, FEVI y el tratamiento farmacológico ${ }^{61}$. Del mismo modo, Anker et $\mathrm{al}^{11}$ informaron que niveles plasmáticos elevados de ácido úrico en pacientes con insuficiencia cardiaca crónica se asociaron a una mayor mortalidad o necesidad de trasplante cardiaco en pacientes con insuficiencia cardiaca crónica, independiente de la función renal, sodio plasmático, uso de diuréticos y edad del paciente. Este estudio intentó establecer al ácido úrico como un marcador de pronóstico y terapéutico adverso en la insuficiencia cardiaca crónica ${ }^{11}$.

Por otro lado, varios estudios clínicos y multicéntricos han abordado el efecto de la inhibición de la XO con alopurinol u oxipurinol en la insuficiencia cardiaca, evaluando aspectos moleculares, clínicos y de morbi-mortalidad (Tabla 1). Por ejemplo, algunos estudios han evaluado la inhibición de $\mathrm{XO}$ respecto a la mejoría en la función endotelial de vasos periféricos. Todos ellos han mostrado una mejoría en la capacidad vasodilatadora tanto local como sistémica. Doehner et al ${ }^{62}$ determinaron mediante la adición de una infusión de acetilcolina y nitroglicerina, la vasodilatación dependiente (VDE) e independiente (VIE) del endotelio, respectivamente, en la arteria radial en 10 pacientes con insuficiencia cardiaca normouricémicos y 9 pacientes hiperuricémicos. En estos 
Tabla 1. Estudios clínicos que evalúan el efecto de la inhibición de xantino oxidasa en pacientes con insuficiencia cardiaca crónica

\begin{tabular}{|c|c|c|c|c|c|}
\hline Autores & Tratamiento & $\begin{array}{l}\text { Dosis } \\
\text { día }\end{array}$ & $\begin{array}{l}\text { Aleatorio } \\
\text { placebo }\end{array}$ & n pacientes & Endpoints \\
\hline Shehab et $a^{88}$ & $\begin{array}{l}\text { Alopurinol } \\
1 \text { semana }\end{array}$ & $\begin{array}{l}100 \mathrm{o} \\
300 \mathrm{mg}\end{array}$ & Sí & 16 & Función autonómica \\
\hline Cappola et al60 & $\begin{array}{l}\text { Alopurinol } \\
45 \mathrm{~min}\end{array}$ & $\begin{array}{l}0.5-1.5 \mathrm{mg} / \mathrm{min} \\
15 \mathrm{~min}\end{array}$ & No & 9 & $\begin{array}{l}\text { Función mecánica y energé- } \\
\text { tica }\end{array}$ \\
\hline Doehner et al ${ }^{62}$ & $\begin{array}{l}\text { Alopurinol } \\
1 \text { semana }\end{array}$ & $\begin{array}{l}300 \circ \\
600 \mathrm{mg} \mu \mathrm{g} / \mathrm{min}\end{array}$ & Sí & $\begin{array}{l}19 \\
14\end{array}$ & Función endotelial \\
\hline Farquharson et al ${ }^{89}$ & $\begin{array}{l}\text { Alopurinol } \\
1 \text { mes }\end{array}$ & $300 \mathrm{mg}$ & Sí & 11 & Función endotelial \\
\hline Gavin et $a^{64}$ & $\begin{array}{l}\text { Alopurinol } \\
3 \text { meses }\end{array}$ & $300 \mathrm{mg}$ & Sí & 50 & BNP y TC6M \\
\hline George et $a^{63}$ & $\begin{array}{l}\text { Alopurinol } \\
\text { Probenecid } \\
1 \text { mes }\end{array}$ & $\begin{array}{l}300-600 \mathrm{mg} \\
1000 \mathrm{mg}\end{array}$ & Sí & $\begin{array}{l}30 \\
26\end{array}$ & $\begin{array}{l}\text { Función } \\
\text { endotelial }\end{array}$ \\
\hline Cingolani et a $\left.\right|^{90}$ & $\begin{array}{l}\text { Oxipurinol } \\
1 \text { mes }\end{array}$ & $600 \mathrm{mg}$ & Sí & 60 & $\begin{array}{l}\text { FE } \\
\text { TC6M }\end{array}$ \\
\hline Hare et al65 & $\begin{array}{l}\text { Oxipurinol } \\
2 \text { años }\end{array}$ & $600 \mathrm{mg}$ & Sí & 405 & $\begin{array}{l}\text { Morbilidad } \\
\text { Mortalidad }\end{array}$ \\
\hline $\begin{array}{l}\text { Greig et al } \\
\text { (Datos no } \\
\text { publicados) }\end{array}$ & $\begin{array}{l}\text { Atorvastatina } \\
\pm \text { Alopurinol } \\
2 \text { meses }\end{array}$ & $\begin{array}{l}20 \mathrm{mg} \\
300 \mathrm{mg}\end{array}$ & Sí & 76 & $\begin{array}{l}\text { Función cardiaca y endotelial } \\
\text { Estrés oxidativo } \\
\text { Inflamación } \\
\text { Remodelado }\end{array}$ \\
\hline
\end{tabular}

últimos, la infusión de alopurinol mejoró la VDE, pero no la VIE. Además, en un estudio cruzado, doble ciego, 14 pacientes hiperuricémicos con insuficiencia cardiaca se distribuyen en forma aleatoria a placebo o alopurinol por 1 semana. Este estudio mostró que los pacientes tratados con alopurinol presentaron una mejoría significativa de la función endotelial y que esta mejoría se correlacionó con el cambio en los niveles de ácido úrico ${ }^{62}$.

Recientemente, dos estudios aleatorizados y controlados con placebo no encontraron diferencias significativas en la mejoría de la capacidad funcional submáxima en este grupo de pacientes. En estos trabajos, tanto alopurinol (300 mg) como oxipurinol $(600 \mathrm{mg})$ no aumentaron significativamente la distancia recorrida en 6 minutos ${ }^{63,64}$. Estos hallazgos, en conjunto a los anteriores, sugieren quela inhibición de la XO, ya sea con alopurinol o oxipurinol puede mejorar la función endotelial, pero no la capacidad funcional.

Por otro lado, el Oxyourinol Theraphy for Congestive Heart Failure Trial ${ }^{65}$ (OPT-CHF) fue el primer estudio clínico, multicéntrico, doble ciego y controlado con placebo, que mostró los efectos de la inhibición de oxipurinol $(600 \mathrm{mg})$ en pacientes con insuficiencia cardiaca sintomática, disfunción sistólica y terapia médica óptima. Este estudio no mostró diferencias significativas en cuanto a morbilidad, mortalidad y calidad de vida, comparado con el placebo, pese a que la terapia con oxipurinol redujo significativamente los niveles de ácido úrico. En el análisis por subgrupos, se observó que los pacientes con ácido úrico basal $>9,5 \mathrm{mg} / \mathrm{dL}(\mathrm{n}=108)$ respondieron favorablemente al oxipurinol, mientras que los pacientes tratados con oxipurinol con niveles $<9,5 \mathrm{mg} / \mathrm{dL}$ exhibieron una tendencia al empeoramiento, siendo esta diferencia no estadísticamente significativa. Además, se observó que en el subgrupo tratado con oxipurinol, la reducción en los niveles de ácido úrico correlacionó favorablemente con la respuesta clínica. Estos autores sugieren que estos resultados son consistentes con los resultados epidemiológicos reportados por Anker et $\mathrm{al}^{11}$, indicando que el 
Rol del ácido úrico en insuficiencia cardiaca - H. Alcaíno et al

ácido úrico puede ser un excelente biomarcador de morbi-mortalidad en la insuficiencia cardiaca.

A pesar de esta amplia información molecular, clínica y epidemiológica, la importancia relativa de esta asociación entre el ácido úrico y la insuficiencia cardiaca sigue siendo controversial. Los autores del estudio Framingham han argumentado que el ácido úrico no es un factor de riesgo de enfermedades cardiovasculares y que en la evaluación del paciente, sólo se debe confiar en los factores de riesgo clásicos ${ }^{66}$. Una dificultad para determinar si el ácido úrico por sí mismo debe ser considerado un factor de riesgo cardiovascular es que la hiperuricemia suele ir asociada con factores de riesgo cardiovascular establecidos y que varios de los estudios que recalcan esta acción deletérea en la insuficiencia cardiaca tienen sesgos de selección de pacientes y tratamiento de datos, entre otros ${ }^{66}$. Por último, con el uso de alopurinol no es posible diferenciar si los efectos benéficos o deletéreos en el sistema cardiovascular son por la reducción de EROs o ácido úrico ${ }^{63}$.

\section{Efectos beneficiosos del ácido úrico en la insuficiencia cardiaca}

\section{Estudios básicos}

Existe una extensa literatura científica in vitro indicando que el ácido úrico posee una fuerte actividad antioxidante ${ }^{67-71}$. Varios mecanismos que han sido descritos pueden explicar en gran medida, su actividad antioxidante en el sistema cardiovascular $25,72,73$.

El estudio publicado por Hink et $\mathrm{al}^{74}$ demostró que tanto in vitro como en animales, el ácido úrico (a concentraciones cercanas a los niveles fisiológicos presentes en humanos), previene la inactivación de superóxido dismutasa extracelular (ecSOD) y SOD citosólica por el peróxido de hidrógeno mediante la reacción de peroxidasa que poseen las isoformas de SOD. Especialmente, en modelos animales se observó que al inhibir la uricasa se encontró que el aumento en los niveles de ácido úrico se relacionaba directamente con una mayor actividad de ecSOD y SOD citosólica en ratas deficientes en apoE, pero no en ratones silvestres. Este resultado sugiere que la inactivación de SOD puede participar en procesos cardiovasculares deletéreos y que el ácido úrico podría preservar esta actividad en humanos. Esta hipótesis nos condujo a demostrar si esta asociación es relevante en humanos. Para este fin, investigamos la asociación entre los niveles de ácido úrico y la actividad de ecSOD y SOD eritrocitaria en humanos ${ }^{25}$. Nuestros resultados muestran una correlación positiva entre los niveles de ácido úrico circulante y la actividad de ecSOD y SOD eritrocitaria en pacientes con insuficiencia cardiaca ${ }^{25}$. Asimismo, Tan et $\mathrm{al}^{75}$ han mostrado in vitro que el ácido úrico a concentraciones de 150 y 300 mM disminuyó la oxidación de xantina a ácido úrico y la formación de anión superóxido en 23 y $32 \%$, respectivamente. Este resultado indica que el ácido úrico es un inhibidor efectivo de la formación de superóxido y de peróxido de hidrógeno por la acción enzimática de la XO a concentraciones cercanas a las encontradas en humanos, reflejando así la compleja interacción entre estrés oxidativo y los sistemas antioxidantes ${ }^{75}$. Adicionalmente, el ácido úrico estabiliza al ácido ascórbico a través en una reacción que no implica degradación de ácido úrico ${ }^{76}$. De hecho, se ha propuesto que el ácido úrico podría ser una adaptación evolutiva a nuestra incapacidad de sintetizar ácido ascórbico, permitiendo la actividad antioxidante del ascorbato en la vasculatura ${ }^{77}$.

En resumen, esta evidencia experimental muestra que en ciertas condiciones tanto in vitro como in vivo la actividad antioxidante del ácido úrico protege al sistema cardiovascular.

\section{Estudios clínicos y epidemiológicos}

La elevación en los niveles plasmáticos de ácido úrico han sido asociados con el incremento de la actividad antioxidante expresado, a través de la medición de la capacidad antioxidante en plasma (FRAP). De hecho, se ha determinado que el ácido úrico entrega $60 \%$ de la capacidad antioxidante total que posee el plasma en humanos ${ }^{24}$. En este contexto, existen investigaciones indicando que la administración previa de ácido úrico a sujetos sanos atenuaba el incremento en los niveles plasmáticos de 8 - iso-PGF2 $\alpha$ (un marcador de estrés oxidativo) que se induce por el ejercicio ${ }^{78}$. Es altamente probable que este efecto sea por su capacidad de contrarrestar la presencia de EROs, debido principalmente a las propiedades antioxidantes del ácido úrico ${ }^{78}$. En otros estudios se ha visto que la administración de L-arginina disminuye los niveles de EROs y que se acompaña temporalmente con un aumento agudo en las concentraciones plasmáticas de ácido úrico ${ }^{79}$. 
Los posibles efectos beneficiosos del ácido úrico en las enfermedades cardiovasculares han sido observados en estudios clínicos multicéntricos. Por ejemplo, en el estudio ALLHAT (Antihypertensive and Lipid-Lowering Treatment to Prevent Heart Attack Trial), el cual incluyó más de 30.000 pacientes hipertensos tratados con clortalidona, se sugirió que la reducción de la excreción renal y, concomitantemente, el aumento plasmático de los niveles de ácido úrico inducidos por el diurético, podría influir positivamente en la sobrevida de estos pacientes, y que concuerda con la hipótesis según lo propuesto por Reyes et al ${ }^{80}$.

\section{La paradoja}

Considerando toda la información existente que relaciona el ácido úrico con la insuficiencia cardiaca, no es posible llegar a un consenso final respecto a las funciones más importantes de esta biomolécula en el sistema cardiovascular. Es claro que, de acuerdo a toda la literatura citada, el ácido úrico posee efectos demostrados tanto deletéreos como también beneficiosos. Creemos que es imposible sostener que el ácido úrico sea per se una molécula dañina o beneficiosa en la insuficiencia cardiaca. En este último tiempo se ha postulado una interesante hipótesis que formula al ácido úrico como una molécula paradójica en el sistema cardiovascular ${ }^{81-83}$. Se han formulado diferentes explicaciones para entender esta dicotomía. Para eso, es necesario circunscribirnos a sus propiedades de temporalidad, físico-químicas, compartimentales y celulares (Tabla 2).

En el último tiempo, se ha postulado que el ácido úrico puede pasar de ser una excelente molécula antioxidante a una fuerte molécula prooxidante. Este cambio puede depender de los sistemas físico-químicos y estructuras químicas que interaccionan con el ácido úrico (Ej: presencia de ácido ascórbico, metales de transición, moléculas pro-oxidantes, etcétera ${ }^{84}$. Adicionalmente, de acuerdo a ciertos estudios in vitro, se ha podido demostrar que este paso de antioxidante a prooxidante del ácido úrico es dependiente, además, del compartimento intracelular o extracelular en donde se encuentre. De hecho, la actividad antioxidante es más eficiente en condiciones hidrofílicas o acuosas que en medios hidrofóbicos ${ }^{83}$. Por esto, es muy probable que a nivel extracelular, como en el plasma sanguíneo, las propiedades antioxidantes del ácido úrico se realicen en condiciones adecuadas, debido a las propiedades de hidrosolubilidad y a la presencia de otras moléculas antioxidantes que son capaces de reciclar al ácido úrico para perpetuar su acción antioxidante. Por el contrario, se ha visto que cuando el ácido úrico pierde estas condiciones fisicoquímicas antes descritas, como en el caso de la entrada a la célula (medio intracelular), o en zonas altamente hidrofóbicas (por ejemplo la placa aterosclerótica y tejido adiposo). En estas zonas, esta molécula adquiere propiedades pro-oxidantes y fomenta la perpetuación del estrés

Tabla 2. Características del ácido úrico en diferentes condiciones moleculares, físico-químicas y celulares

\begin{tabular}{|lll|}
\hline \multicolumn{1}{|c|}{ Antioxidante } & Pro-oxidante \\
\hline Temporalidad & $\begin{array}{l}\text { Aguda } \\
\text { Crónica }\end{array}$ & Crónica \\
\hline Compartimento celular & Hidrosoluble & Liposoluble \\
& Extracelular (plasma) & $\begin{array}{l}\text { Intracelular (Membrana) } \\
\text { Intracelular (citosol) }\end{array}$ \\
Concentración & Placa aterosclerótica \\
\hline Tipo celular & Baja & Alta \\
& Normal & \\
& Alta & Adipocito \\
& Eritrocito & Endotelio vascular \\
\hline
\end{tabular}




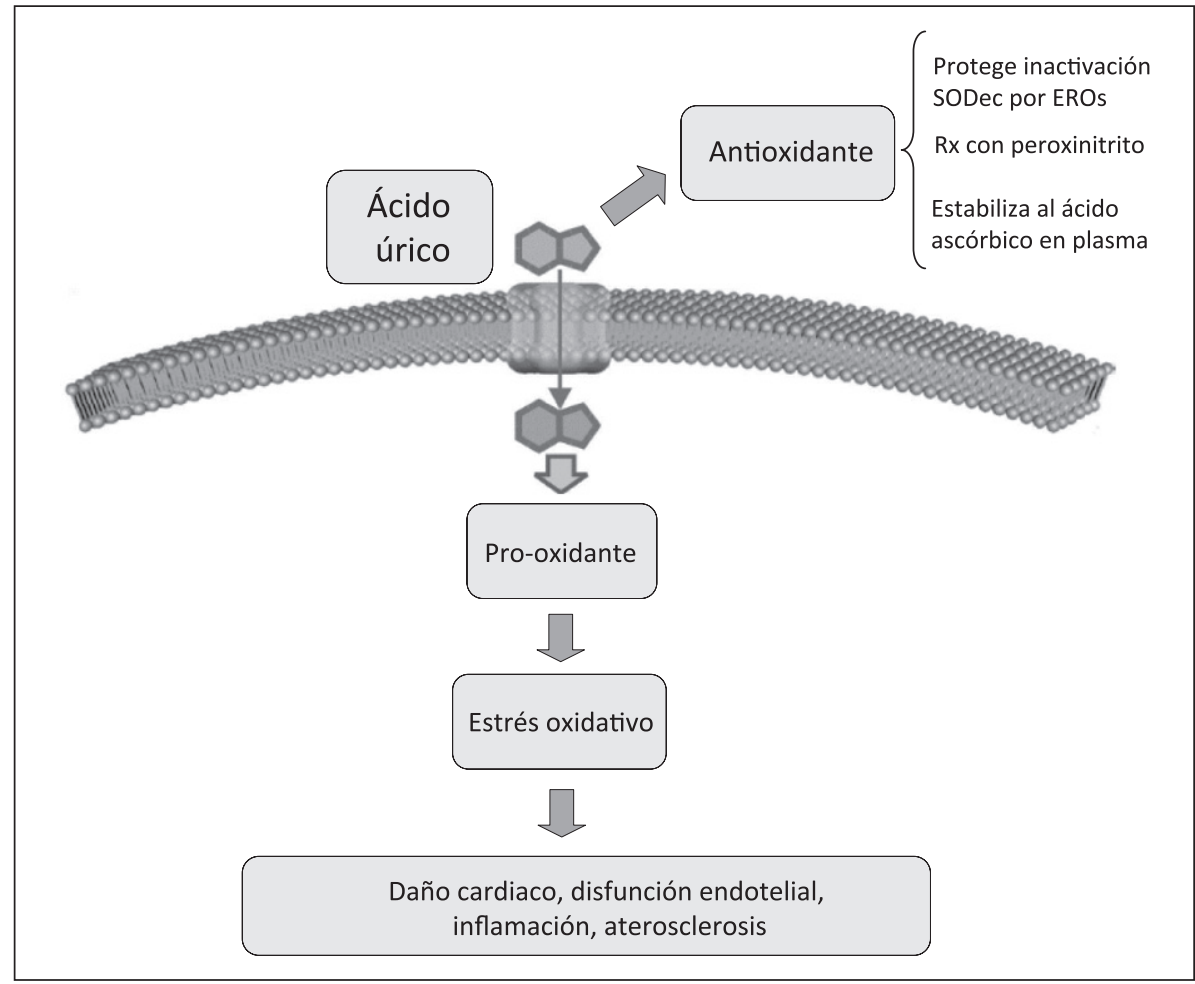

Figura 2. Modelo general de acción antioxidante y pro-oxidante del ácido úrico a nivel celular.

oxidativo en estos compartimentos (Figura 2) $)^{85,86}$. Recientemente, Kim et $\mathrm{al}^{87}$ han implementado una novedosa, sensible y específica técnica analítica (cromatografía líquida acoplada a espectrometría de masa en tándem) con el fin de determinar los niveles de ácido úrico extracelulares e intracelulares en forma independiente ${ }^{87}$. Este interesante trabajo entrega una importante proyección básica y clínica, puesto que la estrategia para estudiar por separado, y muy detalladamente, las funciones del ácido úrico tanto a nivel extracelular como también en forma intracelular; podrá ayudar a resolver la paradoja del ácido úrico a nivel cardiovascular.

\section{Conclusiones}

A pesar de la amplia información molecular, clínica y epidemiológica, la importancia relativa de esta asociación entre el ácido úrico y la insuficiencia cardiaca sigue siendo controvertida. De acuerdo a las evidencias entregadas, es posible sugerir que el ácido úrico no es un buen biomarcador clínico en la insuficiencia cardiaca como se ha propuesto por algunos grupos de investigación, pues su concentración y actividad depende de aspectos tanto moleculares (efecto pro y anti-oxidante), clínicos como demográficos, pudiendo así, explicar en parte la paradójica función del ácido úrico en la fisiopatología de la insuficiencia cardiaca.

\section{Referencias}

1. Castro GP, Verdejo PH, Vukasovic JL, Garcés E, González I. [Predictors of hospital death and prolonged hospitalization in patients with cardiac failure in Chilean hospitals]. Rev Med Chile 2006; 134: 1083-91.

2. Vukasovic JL, Castro GP, Sepúlveda ML, Nazzal NC, Garcés FE, Concepción CR, et al. [Characteristics of heart failure with preserved ejection fraction: results of the Chilean national registry of heart failure, ICARO]. Rev Med Chile 2006; 134: 539-48.

3. Castro P, Vukasovic JL, Garcés E, Sepúlveda L, Ferrada M, Alvarado S. [Cardiac failure in Chilean hospitals: results of the National Registry of Heart Failure, ICARO]. Rev Med Chile 2004; 132: 655-62. 
4. Greig D, Castro P, Gabrielli L, Miranda R, Verdejo H, Alcaino $\mathrm{H}$, et al. [Inflammation and endothelial dysfunction in patients with chronic heart failure]. Rev Med Chile 2008; 136: 687-93.

5. Murray AJ, Edwards LM, Clarke K. Mitochondria and heart failure. Curr Opin Clin Nutr Metab Care 2007; 10: 704-11.

6. Nystrom T. The potential beneficial role of glucagon-like peptide- 1 in endothelial dysfunction and heart failure associated with insulin resistance. Horm Metab Res 2008; 40: 593-606.

7. Rosca MG, Hoppel CL. New aspects of impaired mitochondrial function in heart failure. J Bioenerg Biomembr 2009; 41: 107-12.

8. Lainscak M, von Haehling S, Springer J, Anker SD. Biomarkers for chronic heart failure. Heart Fail Monit 2007; 5: 77-82.

9. Lainscak M, Anker SD. Prognostic factors in chronic heart failure. A review of serum biomarkers, metabolic changes, symptoms, and scoring systems. Herz 2009; 34: 141-7.

10. Hare JM, Johnson RJ. Uric acid predicts clinical outcomes in heart failure: insights regarding the role of xanthine oxidase and uric acid in disease pathophysiology. Circulation 2003; 107: 1951-3.

11. Anker SD, Doehner W, Rauchhaus M, Sharma R, Francis D, Knosalla C, et al. Uric acid and survival in chronic heart failure: validation and application in metabolic, functional, and hemodynamic staging. Circulation 2003; 107: 1991-7.

12. Martínez A, González A, Cerda C, Pérez P, Castro P, Pérez O, et al. [Prognostic value of hyperuricemia in chronic heart failure]. Rev Med Chile 2004; 132: 1031 36.

13. Cengel A, Turkoglu S, Turfan M, Boyaci B. Serum uric acid levels as a predictor of in-hospital death in patients hospitalized for decompensated heart failure. Acta Cardiol 2005; 60: 489-92.

14. Niizeki T, Takeishi Y, Arimoto T, Okuyama H, Nozaki $\mathrm{N}$, Hirono $\mathrm{O}$, et al. Hyperuricemia associated with high cardiac event rates in the elderly with chronic heart failure. J Cardiol 2006; 47: 219-28.

15. Jankowska EA, Ponikowska B, Majda J, Zymlinski R, Trzaska M, Reczuch K, et al. Hyperuricaemia predicts poor outcome in patients with mild to moderate chronic heart failure. Int J Cardiol 2007; 115: 151-5.

16. Sakai H, Tsutamoto T, Tsutsui T, Tanaka T, Ishikawa C, Horie M. Serum level of uric acid, partly secreted from the failing heart, is a prognostic marker in patients with congestive heart failure. Circ J 2006; 70: 1006-11.

17. Doehner W, Springer J, Landmesser U, Struthers AD,
Anker SD. Uric acid in chronic heart failure-current pathophysiological concepts. Eur J Heart Fail 2008; 10: 1269-70.

18. Ekundayo OJ, Dell'italia LJ, Sanders PW, Arnett D, Aban I, Love TE, et al. Association between hyperuricemia and incident heart failure among older adults: A propensitymatched study. Int J Cardiol 2009.

19. Nakanishi N, Suzuki K, Kawashimo H, Nakamura K, Tatara K. Serum uric acid: correlation with biological, clinical and behavioral factors in Japanese men. J Epidemiol 1999; 9: 99-106.

20. Nieto FJ, Iribarren C, Gross MD, Comstock GW, Cutler RG. Uric acid and serum antioxidant capacity: a reaction to atherosclerosis? Atherosclerosis 2000; 148: 131-9.

21. Reyes AJ, Leary WP. The increase in serum uric acid induced by diuretics could be beneficial to cardiovascular prognosis in hypertension: a hypothesis. J Hypertens 2003; 21: 1775-77.

22. Chamorro A, Planas AM, Muner DS, Deulofeu R. Uric acid administration for neuroprotection in patients with acute brain ischemia. Med Hypotheses 2004; 62: 173-6.

23. Hsu CC, Yin MC, Tian R. Ascorbic acid and uric acid suppress glucose-induced fibronectin and vascular endothelial growth factor production in human endothelial cells. J Diabetes Complications 2005; 19: 96-100.

24. Reyes AJ. The increase in serum uric acid concentration caused by diuretics might be beneficial in heart failure. Eur J Heart Fail 2005; 7: 461-67.

25. Alcaíno H, Greig D, Chiong M, Verdejo H, Miranda R, Concepción R, et al. Serum uric acid correlates with extracellular superoxide dismutase activity in patients with chronic heart failure. Eur J Heart Fail 2008; 10: 646-51.

26. Berry CE, Hare JM. Xanthine oxidoreductase and cardiovascular disease: molecular mechanisms and pathophysiological implications. J Physiol 2004; 555: 589-606.

27. Hille R, Massey V. Studies on the oxidative half-reaction of xanthine oxidase. J Biol Chem 1981; 256: 9090-5.

28. Nishino T. The conversion of xanthine dehydrogenase to xanthine oxidase and the role of the enzyme in reperfusion injury. J Biochem 1994; 116: 1-6.

29. Waud WR, Rajagopalan KV. Purification and properties of the NAD+-dependent (type D) and O2-dependent (type O) forms of rat liver xanthine dehydrogenase. Arch Biochem Biophys 1976; 172: 354-64.

30. Della CE, Gozzetti G, Novello F, Stirpe F. Properties of the xanthine oxidase from human liver. Biochim Biophys Acta 1969; 191: 164-6.

31. Linder N, Rapola J, Raivio KO. Cellular expression of xanthine oxidoreductase protein in normal human tissues. Lab Invest 1999; 79: 967-74.

32. de Jong JW, van der MP, Nieukoop AS, Huizer T, Stroeve 
RJ, Bos E. Xanthine oxidoreductase activity in perfused hearts of various species, including humans. Circ Res 1990; 67: 770-3.

33. Sarnesto A, Linder N, Raivio KO. Organ distribution and molecular forms of human xanthine dehydrogenase/ xanthine oxidase protein. Lab Invest 1996; 74: 48-56.

34. Linder N, Rapola J, Raivio KO. Cellular expression of xanthine oxidoreductase protein in normal human tissues. Lab Invest 1999; 79: 967-74.

35. Landmesser U, Spiekermann S, Dikalov S, Tatge H, Wilke $\mathrm{R}$, Kohler C, et al. Vascular oxidative stress and endothelial dysfunction in patients with chronic heart failure: role of xanthine-oxidase and extracellular superoxide dismutase. Circulation 2002; 106: 3073-8.

36. García PJ, Mateos FA. Clinical and biochemical aspects of uric acid overproduction. Pharm World Sci 1994; 16: 40-54.

37. Johnson RJ, Titte S, Cade JR, Rideout BA, Oliver WJ. Uric acid, evolution and primitive cultures. Semin $\mathrm{Ne}$ phrol 2005; 25: 3-8.

38. Seddon M, Looi YH, Shah AM. Oxidative stress and redox signalling in cardiac hypertrophy and heart failure. Heart 2007; 93: 903-7.

39. Shi Y, Evans JE, Rock KL. Molecular identification of a danger signal that alerts the immune system to dying cells. Nature 2003; 425: 516-21.

40. Shi Y, Evans JE, Rock KL. Molecular identification of a danger signal that alerts the immune system to dying cells. Nature 2003; 425: 516-21.

41. Schlotte V, Sevanian A, Hochstein P, Weithmann KU. Effect of uric acid and chemical analogues on oxidation of human low density lipoprotein in vitro. Free Radic Biol Med 1998; 25: 839-47.

42. Alwan WH, Dieppe PA, Elson CJ, Bradfield JW. Hydroxyapatite and urate crystal induced cytokine release by macrophages. Ann Rheum Dis 1989; 48: 476-82.

43. Jaramillo M, Godbout $\mathrm{M}$, Naccache $\mathrm{PH}$, Olivier M. Signaling events involved in macrophage chemokine expression in response to monosodium urate crystals. J Biol Chem 2004; 279: 52797-805.

44. Kanellis J, Watanabe S, Li JH, Kang DH, Li P, Nakagawa $\mathrm{T}$, et al. Uric acid stimulates monocyte chemoattractant protein-1 production in vascular smooth muscle cells via mitogen-activated protein kinase and cyclooxygenase-2. Hypertension 2003; 41: 1287-93.

45. Kang DH, Han L, Ouyang X, Kahn AM, Kanellis J, Li $\mathrm{P}$, et al. Uric acid causes vascular smooth muscle cell proliferation by entering cells via a functional urate transporter. Am J Nephrol 2005; 25: 425-33.

46. Tousoulis D, Charakida M, Stefanadis C. Inflammation and endothelial dysfunction as therapeutic targets in patients with heart failure. Int J Cardiol 2005; 100: 347 53.

47. Miranda HR, Castro GP, Verdejo PH, Chiong M, Díaz-Araya G, Mellado R, et al. [Oxidative stress and inflammation in heart failure: mechanisms of damage and therapeutic alternatives]. Rev Med Chile 2007; 135: 1056-63.

48. Mazzali M, Hughes J, Kim YG, Jefferson JA, Kang DH, Gordon KL, et al. Elevated uric acid increases blood pressure in the rat by a novel crystal-independent mechanism. Hypertension 2001; 38: 1101-6.

49. Khosla UM, Zharikov S, Finch JL, Nakagawa T, Roncal $\mathrm{C}, \mathrm{Mu} \mathrm{W}$, et al. Hyperuricemia induces endothelial dysfunction. Kidney Int 2005; 67: 1739-42.

50. Kang DH, Park SK, Lee IK, Johnson RJ. Uric acidinduced C-reactive protein expression: implication on cell proliferation and nitric oxide production of human vascular cells. J Am Soc Nephrol 2005; 16: 3553-62.

51. de AC, Turner ST, Kullo IJ. Serum uric acid is associated with microvascular function in hypertensive individuals. J Hum Hypertens 2007; 21: 610-5.

52. Langlois M, De Bacquer D, Duprez D, De Buyzere M, Delanghe J, Blaton V. Serum uric acid in hypertensive patients with and without peripheral arterial disease. Atherosclerosis 2003; 168: 163-8.

53. Suzuki I, Yamauchi T, Onuma M, Nozaki S. Allopurinol, an inhibitor of uric acid synthesis-can it be used for the treatment of metabolic syndrome and related disorders? Drugs Today (Barc) 2009; 45: 363-78.

54. Riches PL, Wright AF, Ralston SH. Recent insights into the pathogenesis of hyperuricaemia and gout. Hum Mol Genet 2009; 18: R177-84.

55. Brodov Y, Behar S, Goldenberg I, Boyko V, Chouraqui P. Usefulness of combining serum uric acid and C-reactive protein for risk stratification of patients with coronary artery disease (Bezafibrate Infarction Prevention [BIP] study). Am J Cardiol 2009; 104: 194-8.

56. Brodov Y, Chouraqui P, Goldenberg I, Boyko V, Mandelzweig L, Behar S. Serum uric acid for risk stratification of patients with coronary artery disease. Cardiology 2009; 114: 300-5.

57. Cnossen JS, Ruyter-Hanhijarvi H, van der Post JA, Mol BW, Khan KS, ter Riet G. Accuracy of serum uric acid determination in predicting pre-eclampsia: a systematic review. Acta Obstet Gynecol Scand 2006; 85: 519-25.

58. Feig DI. Uric acid: a novel mediator and marker of risk in chronic kidney disease? Curr Opin Nephrol Hypertens 2009; 18: 526-30.

59. Nakagawa T, Cirillo P, Sato W, Gersch M, Sautin Y, Roncal C, et al. The conundrum of hyperuricemia, metabolic 
syndrome, and renal disease. Intern Emerg Med 2008; 3 : 313-8.

60. Cappola TP, Kass DA, Nelson GS, Berger RD, Rosas GO, Kobeissi ZA, et al. Allopurinol improves myocardial efficiency in patients with idiopathic dilated cardiomyopathy. Circulation 2001; 104: 2407-11.

61. Pascual-Figal DA, Hurtado-Martínez JA, Redondo B, Antolinos MJ, Ruiperez JA, Valdés M. Hyperuricaemia and long-term outcome after hospital discharge in acute heart failure patients. Eur J Heart Fail 2007; 9: 518-24.

62. Doehner W, Schoene N, Rauchhaus M, Leyva-León F, Pavitt DV, Reaveley DA, et al. Effects of xanthine oxidase inhibition with allopurinol on endothelial function and peripheral blood flow in hyperuricemic patients with chronic heart failure: results from 2 placebo-controlled studies. Circulation 2002; 105: 2619-24.

63. George J, Carr E, Davies J, Belch JJ, Struthers A. Highdose allopurinol improves endothelial function by profoundly reducing vascular oxidative stress and not by lowering uric acid. Circulation 2006; 114: 2508-16.

64. Gavin AD, Struthers AD. Allopurinol reduces B-type natriuretic peptide concentrations and haemoglobin but does not alter exercise capacity in chronic heart failure. Heart 2005; 91: 749-53.

65. Hare JM, Mangal B, Brown J, Fisher C, Jr., Freudenberger $\mathrm{R}$, Colucci WS, et al. Impact of oxypurinol in patients with symptomatic heart failure. Results of the OPT-CHF study. J Am Coll Cardiol 2008; 51: 2301-9.

66. Brand FN, McGee DL, Kannel WB, Stokes J, III, Castelli WP. Hyperuricemia as a risk factor of coronary heart disease: The Framingham Study. Am J Epidemiol 1985; 121: 11-8.

67. Meadows J, Smith RC. Uric acid protects erythrocytes from ozone-induced changes. Environ Res 1987; 43: 410-6.

68. Becker BF, Reinholz N, Ozcelik T, Leipert B, Gerlach E. Uric acid as radical scavenger and antioxidant in the heart. Pflugers Arch 1989; 415: 127-35.

69. Souza AV, Petretski JH, Demasi M, Bechara EJ, Oliveira PL. Urate protects a blood-sucking insect against hemininduced oxidative stress. Free Radic Biol Med 1997; 22: 209-14.

70. Gavella M, Lipovac V, Vucic M, Rocic B. Evaluation of ascorbate and urate antioxidant capacity in human semen. Andrologia 1997; 29: 29-35.

71. Spitsin SV, Scott GS, Mikheeva T, Zborek A, Kean RB, Brimer CM, et al. Comparison of uric acid and ascorbic acid in protection against EAE. Free Radic Biol Med 2002; 33: 1363-71.

72. Johnson RJ, Rivard C, Nakagawa T, Sautin YY, SánchezLozada LG. Uric acid: more to learn, more experiments to do. Am J Hypertens 2009; 22: 952-3.

73. Verdejo H, Alcaíno H, Greig D, García L, Pizarro M, Chiong $\mathrm{M}$, et al. Uric acid, xanthine oxidase and heart failure: unresolved issues. Eur J Heart Fail 2008; 10: 1271-2.

74. Hink HU, Santanam N, Dikalov S, McCann L, Nguyen AD, Parthasarathy $S$, et al. Peroxidase properties of extracellular superoxide dismutase: role of uric acid in modulating in vivo activity. Arterioscler Thromb Vasc Biol 2002; 22: 1402-8.

75. Tan S, Radi R, Gaudier F, Evans RA, Rivera A, Kirk KA, et al. Physiologic levels of uric acid inhibit xanthine oxidase in human plasma. Pediatr Res 1993; 34: 303-7.

76. Sevanian A, Davies KJ, Hochstein P. Serum urate as an antioxidant for ascorbic acid. Am J Clin Nutr 1991; 54: 1129S-34S.

77. Johnson RJ, Gaucher EA, Sautin YY, Henderson GN, Angerhofer AJ, Benner SA. The planetary biology of ascorbate and uric acid and their relationship with the epidemic of obesity and cardiovascular disease. Med Hypotheses 2008; 71: 22-31.

78. Waring WS, Convery A, Mishra V, Shenkin A, Webb DJ, Maxwell SR. Uric acid reduces exercise-induced oxidative stress in healthy adults. Clin Sci (Lond) 2003; 105: 425-30.

79. Lin WT, Yang SC, Tsai SC, Huang CC, Lee NY. L-Arginine attenuates xanthine oxidase and myeloperoxidase activities in hearts of rats during exhaustive exercise. $\mathrm{Br}$ J Nutr 2006; 95: 67-75.

80. Reyes AJ, Leary WP. The ALLHAT and the cardioprotection conferred by diuretics in hypertensive patients: a connection with uric acid? Cardiovasc Drugs Ther 2002; 16: 485-7.

81. Lippi G, Montagnana M, Franchini M, Favaloro EJ, Targher G. The paradoxical relationship between serum uric acid and cardiovascular disease. Clin Chim Acta 2008; 392: 1-7.

82. Mandell BF. The crystal, the gout, and the paradox. Cleve Clin J Med 2002; 69: 720.

83. Sautin YY, Johnson RJ. Uric acid: the oxidant-antioxidant paradox. Nucleosides Nucleotides Nucleic Acids 2008; 27: 608-19.

84. Patterson RA, Horsley ET, Leake DS. Prooxidant and antioxidant properties of human serum ultrafiltrates toward LDL: important role of uric acid. J Lipid Res 2003; 44: 512-21.

85. Muraoka S, Miura T. Inhibition by uric acid of free radicals that damage biological molecules. Pharmacol Toxicol 2003; 93: 284-9.

86. Felici C, Ciari I, Terzuoli L, Porcelli B, Setacci C, Giubbolini $\mathrm{M}$, et al. Purine catabolism in advanced carotid 
Rol del ácido úrico en insuficiencia cardiaca - H. Alcaíno et al

artery plaque. Nucleosides Nucleotides Nucleic Acids 2006; 25: 1291-4.

87. Kim KM, Henderson GN, Ouyang X, Frye RF, Sautin YY, Feig DI, et al. A sensitive and specific liquid chromatography-tandem mass spectrometry method for the determination of intracellular and extracellular uric acid. J Chromatogr B Analyt Technol Biomed Life Sci 2009; 877: 2032-8.

88. Shehab AM, Butler R, MacFadyen RJ, Struthers AD. A placebo-controlled study examining the effect of allopu- rinol on heart rate variability and dysrhythmia counts in chronic heart failure. Br J Clin Pharmacol 2001; 51: 329-34.

89. Farquharson CA, Butler R, Hill A, Belch JJ, Struthers AD. Allopurinol improves endothelial dysfunction in chronic heart failure. Circulation 2002; 106: 221-6.

90. Cingolani HE, Plastino JA, Escudero EM, Mangal B, Brown J, Pérez NG. The effect of xanthine oxidase inhibition upon ejection fraction in heart failure patients: La Plata Study. J Card Fail 2006; 12: 491-8. 